\title{
Path Tracking in Cable Suspended Parallel Robots through Position- Dependent Model Predictive Control with Embedded Integrator
}

\author{
$\underline{\text { Jason Bettega }}^{1}$, Dario Richiedei $^{1}$, Alberto Trevisani ${ }^{1}$ \\ ${ }^{1}$ Department of Management and Engineering \\ Università degli Studi di Padova \\ Stradella S. Nicola 3, 36100 Vicenza, Italy \\ jason.bettega@phd.unipd.it \\ [dario.richiedei, alberto.trevisani]@unipd.it
}

\begin{abstract}
This work proposes a novel Model Predictive Control (MPC) algorithm for CableDriven Robots (CDRs), with a suspended configuration, to achieve good performances in terms of trajectory tracking of the end-effector as well as ensuring the positiveness of cable tensions. A two-stage controller is exploited to handle model nonlinearities. Firstly, a position-dependent MPC algorithm with embedded integrator is designed to evaluate the optimal cable tensions that are required to track trajectory, while considering constraints on the feasible tensions. Secondly, the related motor torques are evaluated taking into account the dynamics of the electric motor themselves, through a dedicated feedforward approach. With the goal to assess the proposed control algorithm, a 3-dof cable suspended spatial robot is considered and different trajectory tracking tasks are performed: firstly, an unfeasible reference is tested in order to assess the control algorithm in the worstcase scenario and, secondly, two feasible and common trajectories are considered as reference laws of motion. Simulative outcomes are displayed and contour errors are reported to numerically evaluate the controller performances.
\end{abstract}

Keywords: Model Predictive Control, embedded integrator, Cable-Driven Robots, Trajectory tracking, Tension control.

\section{INTRODUCTION}

Precise path tracking control in Cable Suspended Parallel Robots (CSPRs) is a challenging topic in the field of control of multibody systems, due to the positivity constraints on cable tensions, and for this reason it is attracting even more attention in the literature. On the one hand, from the open-loop point of view, trajectory planning strategies have been suggested to a-priori ensure positive and bounded cable tensions along given paths (e.g. [1], [2]). On the other hand, from the closed-loop perspective, some standard industrial controllers, such as PID controllers, have been often applied to this kind of robotic systems in the last decades, showing adequate results ([3], [4]). However, advanced control techniques have to be considered to get even better performances. Additionally, standard techniques do not embed the positiveness constraints on the cable tensions, as well as bounds on the feasible maximum tensions: since control must complies with these requirements, a-posteriori verification or control saturation are therefore usually adopted.

In this paper, precise path tracking control in a CSPR is solved by exploiting and extending the idea of Model Predictive Control (MPC). Indeed, among the model-based control techniques, MPC has several features that make it very attractive for Cable-Driven Robots (CDRs) in general. The basic idea of MPC is to solve an optimal control problem defined by a cost function over a receding horizon and constrained by the system dynamics and by bounds on 
some variables; these features make it attractive for motion control of multibody systems (see e.g. [5], [6], [7]). The optimization problem over the future control variables is solved at each time step by predicting the future system states and outputs. Hence, MPC provides an optimal sequence of the control input, in accordance with some metrics. The great advantage that makes MPC particularly suitable for CDRs is represented by its capability to embed constraints on input and output variables without requiring a-posteriori saturations.

In the very last years, attention has been spent by a few researchers in the field of CDRs to apply the concepts of MPC ([8], [9], [10], [11], [12]), also showing promising results. Different MPC architectures are proposed by these papers, with reference of fully constrained CDRs, and different approaches are proposed to deal with the nonlinear dynamic behavior of the system. The idea of MPC has been also adopted to plan dynamic transition trajectories for a fullyactuated three-degree-of-freedom cable suspended robot in [13].

In this work, the proposed MPC scheme is based on a two-step strategy that splits the system into two subsystems and uses them in a sequential approach. First, feedback MPC is designed for the subsystem made by the suspended mass by computing the positive tensions. In this way, the dynamic matrix of the state-space model is constant, while just the input matrix depends on the system pose. Nonlinearities is handled by updating the model at each time step of the control loop, and then assuming the input matrix as constant along the prediction interval used for the MPC design, thus reducing the computational burden, and allowing for real-time calculation. Then, a model-based approach is used to compute the reference motor torques, by exploiting the dynamic model of the motors, the optimal tensions computed by the MPC and the commanded speed and acceleration. The control assumes feedback of position and speed of the suspended mass and rigid cables. No tension feedback is instead adopted. To ensure effective tracking, an embedded integrator is here adopted in the MPC formulation (MPC-EI) by formulating the model through the difference variables, that easily allows including the requirement of small variations of the cable tensions to get smooth control actions.

Numerical assessment of the control performances is made through a fully-actuated threedegree-of-freedom cable-suspended robot (i.e. with a lumped end-effector), controlled by three cables.

\section{SYSTEM MODEL}

The studied system is depicted in Figure 1. The subsystem made by the lumped mass suspended through three cables is modeled through three ordinary differential equations (ODEs):

$$
m \ddot{\mathbf{p}}=m \mathbf{g}+\sum_{i=1}^{3}\left(-T_{i} \frac{\mathbf{p}-\mathbf{A}_{i}}{\left\|\mathbf{p}-\mathbf{A}_{i}\right\|}\right) .
$$

$\mathbf{p}=\left[\begin{array}{lll}x & y & z\end{array}\right]^{T} \in \mathbb{R}^{3}$ is the absolute position of the end-effector, whose mass is $\mathrm{m} ; \mathbf{A}_{i} \in \mathbb{R}^{3}$ denotes the absolute position of the fixed output points of the spools $(i=1,2,3)$; $\mathbf{g}=\left[\begin{array}{lll}0 & 0 & -g\end{array}\right]^{T} \in \mathbb{R}^{3}$ is the vector of gravity acceleration; $T_{i}$ is the tension of the $i^{\text {th }}$ cable.

The dynamic model of the $i^{\text {th }}$ motor is described by the following ODE:

$$
J_{i} \ddot{\theta}_{i}(t)+f_{v, i} \dot{\theta}_{i}(t)=C_{m, i}(t)-T_{i}(t) r_{i},
$$

where $\theta_{i}$ is the motor shaft absolute rotation, $J_{i}$ indicates the moment of inertia of rotor, drum and idle pulleys, $f_{v, i}$ is the viscous friction coefficient, $C_{m, i}$ is the motor torque and $r_{i}$ represents the drum radius.

The kinematic constraint equation relating the rotation of the $i^{\text {th }}$ motor and the length of the $i^{\text {th }}$ cable, under the assumption that cables are not slack and therefore behave as holonomic ideal constraints, is: 


$$
\rho_{i}=\rho_{i 0}-r_{i} \theta_{i},
$$

where $\rho_{i}$ is the $i^{\text {th }}$ cable length $\left(\rho_{i}=\left\|\mathbf{p}-\mathbf{A}_{i}\right\|\right)$ and $\rho_{i 0}$ is the cable length corresponding to $\theta_{i}=0$.

Denoting with $\boldsymbol{\theta}=\left[\begin{array}{lll}\theta_{1} & \theta_{2} & \theta_{3}\end{array}\right]^{T} \in \mathbb{R}^{3}$ the vector containing the absolute motor rotations, the resulting dynamic model, in terms of non-minimal coordinates, is defined as follows:

$$
\mathbf{M} \ddot{\mathbf{q}}+\mathbf{J}^{\mathrm{T}} \boldsymbol{\lambda}=\mathbf{Q},
$$

where $\mathbf{M}=\operatorname{diag}\left\{J_{1}, J_{2}, J_{3}, m, m, m\right\} \in \mathbb{R}^{6 \times 6}$ is the mass matrix, $\mathbf{q}=\left[\begin{array}{cc}\mathbf{p}^{T} & \mathbf{\theta}^{T}\end{array}\right]^{T} \in \mathbb{R}^{6}$ is a set of six dependent coordinates containing both the absolute cartesian position of the end-effector $\mathbf{p}$ and the absolute motor rotations $\boldsymbol{\theta}, \mathbf{J} \in \mathbb{R}^{6 \times 3}$ is the Jacobian of the position constraints, $\lambda \in$ $\mathbb{R}^{3 \times 1}$ is the vector of the Lagrange multipliers and $\mathbf{Q} \in \mathbb{R}^{6 \times 1}$ contains the external forces (gravity forces, friction, motor torques).

To meet the formalism of control theory, the set of DAEs obtained is converted into a minimal set of ODEs. By exploiting a matrix $\mathbf{R} \in \mathbb{R}^{6 \times 3}$ such that

$$
\dot{\mathbf{q}}=\mathbf{R} \dot{\mathbf{p}},
$$

then the usual form of a multibody system dynamic model is obtained:

$$
\left(\mathbf{R}^{\mathrm{T}} \mathbf{M} \mathbf{R}\right) \ddot{\mathbf{p}}+\left(\mathbf{R}^{\mathrm{T}} \mathbf{M} \dot{\mathbf{R}}\right) \dot{\mathbf{p}}=\mathbf{R}^{\mathrm{T}} \mathbf{Q} .
$$

Since Eq. (6) directly relates the input torques of the electric motors (contained in vector $\mathbf{Q}$ ) with the end-effector cartesian position, such a model is exploited to simulate the real system in a Matlab-Simulink environment, also including simplified models of the sensors and the actuators.

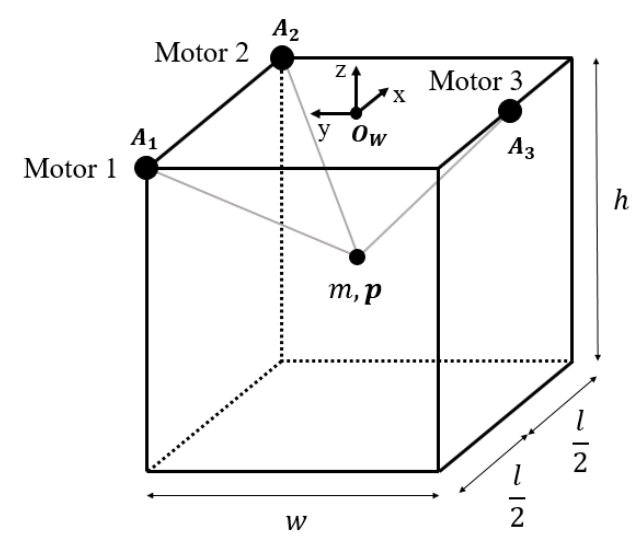

Figure 1. Scheme of the CSPR under investigation.

\section{DESIGN OF THE CONTROL SCHEME}

The dynamic model in Eq. (6) is nonlinear, due to the dependence of $\mathbf{R}$ on positions. To simplify the control design, the synthesis of the proposed MPC scheme is based on a two-step strategy that splits the system into two subsystems and uses them in a sequential approach. First, feedback MPC is designed for the subsystem made by the suspended mass by computing the positive tensions to track the desired spatial path. Then, the model in Eq. (2) is adopted to compute the reference motor torques. Further feedforward terms compensating for gravity forces and inertial terms of the suspended mass could be adopted to improve; however, in this work they are not included in order to emphasize the effectiveness of the feedback action.

\subsection{Synthesis of MPC}


State-Space model. The ODEs in Eq. (1) can be transformed into a first-order model through state vector $\chi_{\mathbf{c}}=\left[\begin{array}{ll}\dot{\mathbf{p}}^{T} & \mathbf{p}^{T}\end{array}\right]^{T} \in \mathbb{R}^{6}$, obtaining the following nonlinear continuous-time statespace system:

$$
\left\{\begin{array}{l}
\dot{\chi}_{\mathbf{c}}(t)=\mathbf{A}_{\mathbf{c}} \chi_{\mathbf{c}}(t)+\mathbf{B}_{\mathbf{c}}(\mathbf{p}) \mathbf{T}(t)+\mathbf{B}_{\mathbf{g c}} \mathbf{g} \\
\mathbf{y}_{\mathbf{c}}(t)=\mathbf{C}_{\mathbf{c}} \boldsymbol{\chi}_{\mathbf{c}}(t)
\end{array},\right.
$$

where $\mathbf{A}_{\mathbf{c}} \in \mathbb{R}^{6 \times 6}$ is the constant dynamic matrix, $\mathbf{B}_{\mathbf{c}}(\mathbf{p}) \in \mathbb{R}^{6 \times 3}$ is the pose-dependent input matrix, $\mathbf{B}_{\mathbf{g c}} \in \mathbb{R}^{6 \times 3}$ is the matrix that describe the relationship between the vector of gravity acceleration and the state vector, $\mathbf{C}_{\mathbf{c}} \in \mathbb{R}^{3 \times 6}$ is the output matrix, and are defined as follows ( $\mathbf{V}(\mathbf{p}) \in \mathbb{R}^{3 \times 3}$ is the matrix containing the unitary vectors of the cables, $\mathbf{I}_{3} \in \mathbb{R}^{3 \times 3}$ is the identity matrix and $\mathbf{0}_{3} \in \mathbb{R}^{3 \times 3}$ is the null matrix):

$$
\mathbf{A}_{\mathbf{c}}=\left[\begin{array}{ll}
\mathbf{0}_{3} & \mathbf{0}_{3} \\
\mathbf{I}_{3} & \mathbf{0}_{3}
\end{array}\right], \quad \mathbf{B}_{\mathbf{c}}(\mathbf{p})=\left[\begin{array}{c}
-\frac{1}{m} \mathbf{V}(\mathbf{p}) \\
\mathbf{0}_{3}
\end{array}\right], \quad \mathbf{B}_{\mathbf{g c}}=\left[\begin{array}{l}
\mathbf{I}_{3} \\
\mathbf{0}_{3}
\end{array}\right], \quad \mathbf{C}_{\mathbf{c}}=\left[\begin{array}{ll}
\mathbf{0}_{3} & \mathbf{I}_{3}
\end{array}\right] .
$$

The cable tensions are included in the input vector $\mathbf{T}=\left[\begin{array}{lll}T_{1} & T_{2} & T_{3}\end{array}\right]^{T} \in \mathbb{R}^{3}$, and $\mathbf{y}_{\mathbf{c}} \in \mathbb{R}^{3}$ is the system output.

By discretizing the continuous-time state-space model in Eq. (7) with a sampling time $T_{s}$, the following set of difference equations is obtained:

$$
\left\{\begin{aligned}
\boldsymbol{\chi}_{\mathbf{d}}(k+1) & =\mathbf{A}_{\mathbf{d}} \boldsymbol{\chi}_{\mathbf{d}}(k)+\mathbf{B}_{\mathbf{d}}(\mathbf{p}) \mathbf{T}(k)+\mathbf{B}_{\mathbf{g d}} \mathbf{g}, \\
\mathbf{y}_{\mathbf{d}}(k) & =\mathbf{C}_{\mathbf{d}} \boldsymbol{\chi}_{\mathbf{d}}(k)
\end{aligned}\right.
$$

where $k$ denotes the generic time instant, $\boldsymbol{\chi}_{\mathbf{d}}$ and $\mathbf{y}_{\mathbf{d}}$ are, respectively, the discrete state and output vectors, while $\mathbf{A}_{\mathbf{d}} \in \mathbb{R}^{6 \times 6}, \mathbf{B}_{\mathbf{d}}(\mathbf{p}) \in \mathbb{R}^{6 \times 3}, \mathbf{B}_{\mathbf{g d}} \in \mathbb{R}^{6 \times 3}, \mathbf{C}_{\mathbf{d}} \in \mathbb{R}^{3 \times 6}$ are the matrices of the discrete-time model. In this paper, to simplify the computation, discretization is performed through through the following approximation of the Euler method, altough other approaches can be adopted:

$$
\mathbf{A}_{\mathbf{d}}=\mathbf{I}+T_{s} \mathbf{A}_{\mathrm{c}}, \quad \mathbf{B}_{\mathbf{d}}(\mathbf{p})=T_{s} \mathbf{B}_{\mathbf{c}}(\mathbf{p}), \quad \mathbf{B}_{\mathrm{gd}}=T_{s} \mathbf{B}_{\mathrm{gc}}, \quad \mathbf{C}_{\mathbf{d}}=\mathbf{C}_{\mathbf{c}} .
$$

As stated in the Introduction, to provide effective tracking of the spatial path, the formulation of MPC-EI is adopted. Therefore, by introducing the difference variables as follows:

$$
\begin{gathered}
\Delta \boldsymbol{\chi}_{\mathbf{d}}(k)=\boldsymbol{\chi}_{\mathbf{d}}(k)-\boldsymbol{\chi}_{\mathbf{d}}(k-1), \\
\Delta \mathbf{T}(k)=\mathbf{T}(k)-\mathbf{T}(k-1),
\end{gathered}
$$

and the augmented state vector $\chi \in \mathbb{R}^{9}$,

$$
\boldsymbol{\chi}(k)=\left[\begin{array}{c}
\Delta \boldsymbol{\chi}_{\mathbf{d}}(k) \\
\mathbf{y}_{\mathbf{d}}(k)
\end{array}\right],
$$

then the following augmented state-space model is obtained:

$$
\left\{\begin{array}{rl}
\boldsymbol{\chi}(k+1) & =\mathbf{A} \boldsymbol{\chi}(k)+\mathbf{B}(\mathbf{p}) \mathbf{\Delta} \mathbf{T}(k) \\
\mathbf{y}(k) & =\mathbf{C} \boldsymbol{\chi}(k)
\end{array},\right.
$$

where the matrices $\mathbf{A} \in \mathbb{R}^{9 \times 9}, \mathbf{B}(\mathbf{p}) \in \mathbb{R}^{9 \times 3}, \mathbf{C} \in \mathbb{R}^{3 \times 9}$ assume the following meaning:

$$
\mathbf{A}=\left[\begin{array}{cc}
\mathbf{A}_{\mathbf{d}} & \mathbf{0}_{6 \times 3} \\
\mathbf{C}_{\mathbf{d}} \mathbf{A}_{\mathbf{d}} & \mathbf{I}_{3}
\end{array}\right], \quad \mathbf{B}(\mathbf{p})=\left[\begin{array}{c}
\mathbf{B}_{\mathbf{d}}(\mathbf{p}) \\
\mathbf{C}_{\mathbf{d}} \mathbf{B}_{\mathbf{d}}(\mathbf{p})
\end{array}\right], \quad \mathbf{C}=\left[\begin{array}{ll}
\mathbf{0}_{3 \times 6} & \mathbf{I}_{3}
\end{array}\right]
$$

While $\mathbf{A}$ and $\mathbf{C}$ are constant, matrix $\mathbf{B}(\mathbf{p})$ is position dependent. 
Evaluation of the prediction matrices. MPC exploits the output prediction over a selected prediction horizon $N_{p}$. Nonlinearities is handled in this work by updating matrix $\mathbf{B}_{\mathbf{d}}(\mathbf{p})$ at each time step, based on the system configuration. Such a matrix is then assumed constant over the prediction horizon for control design.

The predicted output $\mathbf{Y} \in \mathbb{R}^{3 N_{p}}$ in vectorial form is described as:

$$
\mathbf{Y}=\mathbf{F x}\left(k_{i}\right)+\boldsymbol{\Phi}(\mathbf{p}) \Delta \mathbf{T}_{\mathbf{N}_{\mathbf{c}}},
$$

where the matrices $\mathbf{F} \in \mathbb{R}^{3 N_{p} \times 9}$ and $\boldsymbol{\Phi}(\mathbf{p}) \in \mathbb{R}^{3 N_{p} \times 3 N_{c}}$ assume the following expression:

$$
\mathbf{F}=\left[\begin{array}{c}
\mathbf{C A} \\
\mathbf{C A}^{2} \\
\vdots \\
\mathbf{C A}^{N_{p}}
\end{array}\right], \quad \boldsymbol{\Phi}(\mathbf{p})=\left[\begin{array}{cccc}
\mathbf{C B}(\mathbf{p}) & \mathbf{0} & \cdots & \mathbf{0} \\
\mathbf{C A B}(\mathbf{p}) & \mathbf{C B}(\mathbf{p}) & \cdots & \mathbf{0} \\
\vdots & \vdots & \vdots & \vdots \\
\mathbf{C A}^{N_{p}-1} \mathbf{B}(\mathbf{p}) & \mathbf{C A}^{N_{p}-2} \mathbf{B}(\mathbf{p}) & \cdots & \mathbf{C A}^{N_{p}-N_{c}} \mathbf{B}(\mathbf{p})
\end{array}\right]
$$

Once the value of the state at a generic time instant $k_{i}$ is known, together with the control action along the control horizon, these matrices allow to achieve the value of the outputs along all the prediction horizon, hence describing the future system response.

Constrained optimization problem. The following cost function $J$ is defined and minimized to solve the trajectory tracking problem:

$$
J=\left(\mathbf{Y}^{\text {des }}-\mathbf{Y}\right)^{T} \mathbf{R}_{\mathbf{Y}}\left(\mathbf{Y}^{\text {des }}-\mathbf{Y}\right)+\Delta \mathbf{T}_{\mathbf{N}_{\mathbf{c}}}{ }^{T} \mathbf{R}_{\Delta \mathbf{T}} \Delta \mathbf{T}_{\mathbf{N}_{\mathbf{c}}},
$$

where $\mathbf{R}_{\mathbf{Y}} \in \mathbb{R}^{3 N_{p} \times 3 N_{p}}$ and $\mathbf{R}_{\Delta \mathbf{T}} \in \mathbb{R}^{3 N_{c} \times 3 N_{c}}$ are weighting matrices, $\Delta \mathbf{T}_{\mathbf{N}_{\mathbf{c}}} \in \mathbb{R}^{3 N_{c}}$ contains the future control actions and $\mathbf{Y}^{\text {des }} \in \mathbb{R}^{3 N_{p}}$ is the vector of the reference trajectories along the prediction horizon, described as:

$$
\mathbf{Y}^{\text {des }}=\left[\begin{array}{llll}
\mathbf{I}_{3} & \mathbf{I}_{3} & \cdots & \mathbf{I}_{3}
\end{array}\right]^{T} \mathbf{r}\left(k_{i}\right)=\mathbf{F}^{\mathrm{des}} \mathbf{r}\left(k_{i}\right),
$$

where $\mathbf{F}^{\text {des }} \in \mathbb{R}^{3 N_{p} \times 3}$ and $\mathbf{r}\left(k_{i}\right) \in \mathbb{R}^{3}$ is the vector of reference trajectories at time step $k_{i}$. It should be noted that the proposed formulation of MPC with embedded integrator includes in the cost function the variation of the cable tensions through $\Delta \mathbf{T}_{\mathbf{N}_{\mathrm{c}}}$, rather than the value of the tensions. This aspect is particularly useful in real case scenarios, as discussed in [12], because large variations of cable tensions are not physically feasible.

Constraints on the magnitude of the cable tensions are embedded in the control synthesis through lower $\left(\mathbf{T}_{\min }=T_{\min }\left[\begin{array}{lll}1 & 1 & 1\end{array}\right]^{T}\right)$ and upper bounds $\left(\mathbf{T}_{\max }=T_{\max }\left[\begin{array}{lll}1 & 1 & 1\end{array}\right]^{T}\right)$ on the feasible control input $\mathbf{T}(k)$ defined through the following element-wise inequalities, related to the minimum and the maximum allowable cable tensions $T_{\min }$ and $T_{\max }$ :

$$
\mathbf{T}_{\min } \leq \mathbf{T}(k) \leq \mathbf{T}_{\max } .
$$

Lower bounds define the positiveness tension requirements; upper bounds are related to the maximum admissible load. Constraints are then properly translated into bounds on the difference control signal $\Delta \mathbf{T}(k)$ and hence on $\Delta \mathbf{T}_{\mathbf{N}_{\mathbf{c}}}$. Since $J$ is quadratic and the constraints are described through linear inequalities, a standard quadratic programming problem is adopted to control design. In this paper, the Hildreth's method is exploited because of its good numerical conditioning.

Since the input of the MPC formulation with embedded integrator is defined by the difference variable $\Delta \mathbf{T}(k)$, the predicted optimal inputs are written in a vectorial form as follows: 


$$
\Delta \mathbf{T}_{\mathbf{N}_{\mathbf{c}}}=\left[\begin{array}{llll}
\Delta \mathbf{T}\left(k_{i}\right) & \Delta \mathbf{T}\left(k_{i}+1\right) & \cdots & \Delta \mathbf{T}\left(k_{i}+N_{c}-1\right)
\end{array}\right]^{T},
$$

where $N_{c}$ is the control horizon and it indicates the number of samples that are considered in order to apply the optimal control action. Among all the values contained in the optimal solution $\Delta \mathbf{T}_{\mathbf{N}_{\mathbf{c}}}$, only the first 3 terms (which represent the entries of $\Delta \mathbf{T}\left(k_{i}\right)$ in Eq. (21)) are considered while the remaining ones are discarded, in accordance with the Receding Horizon Principle and therefore making the MPC algorithm a closed-loop controller.

\subsection{Computation of the motor torques}

Once the optimal tensions $T_{i}^{M P C}$ are computed by the proposed MPC, the motor torques $C_{m, i}$ are achieved through a feedforward approach by exploiting the inverse-dynamic model of each actuator (see Eq. (2)), with nominal inertia $\left(\hat{J}_{i}\right)$ and viscous friction coefficients $\left(\hat{f}_{v, i}\right)$, and considering acceleration and speed references $\ddot{\theta}_{i}^{\text {ref }}(t)$ and $\dot{\theta}_{i}^{\text {ref }}(t)$ (obtained through inverse kinematics of the load reference trajectory). Therefore, considering the $i^{\text {th }}$ electric motor, its commanded torque is evaluated as follows:

$$
C_{m, i}=\hat{J}_{i} \ddot{\theta}_{i}^{r e f}(t)+\hat{f}_{v, i} \dot{\theta}_{i}^{r e f}(t)+r_{i} T_{i}^{M P C}(t)
$$

\section{NUMERICAL RESULTS}

\subsection{System description}

The parameters of the studied system are reported in Table 1. In the practical implementation, since MPC is a model-based control technique, it is assumed that accurate tuning of the model parameters is available, by exploiting any of the well-established techniques for model identification in multibody systems (see e.g. [14]), or specifically developed for cable robots (see e.g. [15]). Three motion references have been simulated to highlight the paramount features of the proposed control scheme. A simulation environment has been developed through MatlabSimulink.

Table 1. System parameters

\begin{tabular}{|c|c|c|}
\hline Parameter & Description & Value \\
\hline$J_{m, 1}, J_{m, 2}, J_{m, 3}$ & Motor inertias & $2.6 \times 10^{-5}\left[\mathrm{kgm}^{2}\right]$ \\
\hline$f_{v, 1}, f_{v, 2}, f_{v, 3}$ & Motor viscous friction coefficients & $5 \times 10^{-3}[\mathrm{Nms} / \mathrm{rad}]$ \\
\hline$r_{1}, r_{2}, r_{3}$ & Radius of the pulleys & $0.036[\mathrm{~m}]$ \\
\hline$m$ & Mass of the suspended load & $2.94[\mathrm{~kg}]$ \\
\hline$T_{\min } ; T_{\max }$ & Minimum and maximum tensions & $5 ; 100[\mathrm{~N}]$ \\
\hline$T_{s}$ & Sampling time & $2 \times 10^{-3}[\mathrm{~s}]$ \\
\hline$N_{c} ; N_{p}$ & Control and prediction horizons & $1 ; 60$ \\
\hline $\mathbf{R}_{\mathbf{Y}} ; \mathbf{R}_{\Delta \mathbf{T}}$ & Weighting matrices & $\mathbf{I}_{180} ; 1 \times 10^{-3} \mathbf{I}_{3}$ \\
\hline
\end{tabular}

\subsection{Test cases}

\subsubsection{Test 1: point-to-point motion with an unfeasible trajectory.}

The first trajectory is made by a descending step reference for the z-axis, while keeping the references on $\mathrm{x}$-axis and $\mathrm{y}$-axis equal to the initial conditions. This trajectory is unfeasible be- 
cause it would require negative infinite acceleration along the z-axis as well as negative tensions and hence would lead to slack cables. The presence of constraints on the feasible tensions in the control synthesis allows tracking the reference as fast as possible, while ensuring feasible tensions. The temporal tracking response in the z-axis is displayed in Figure 2, while Figure 3 shows the commanded cable tensions and corroborate the correctness of the proposed approach in handling constraints.

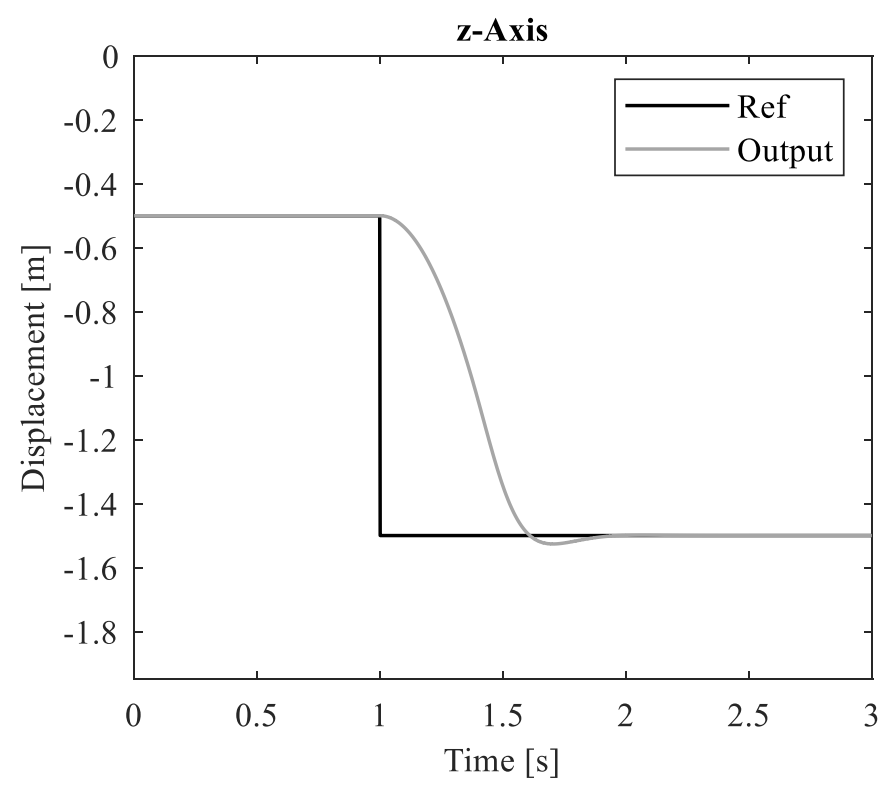

Figure 2. Temporal tracking response with step reference.

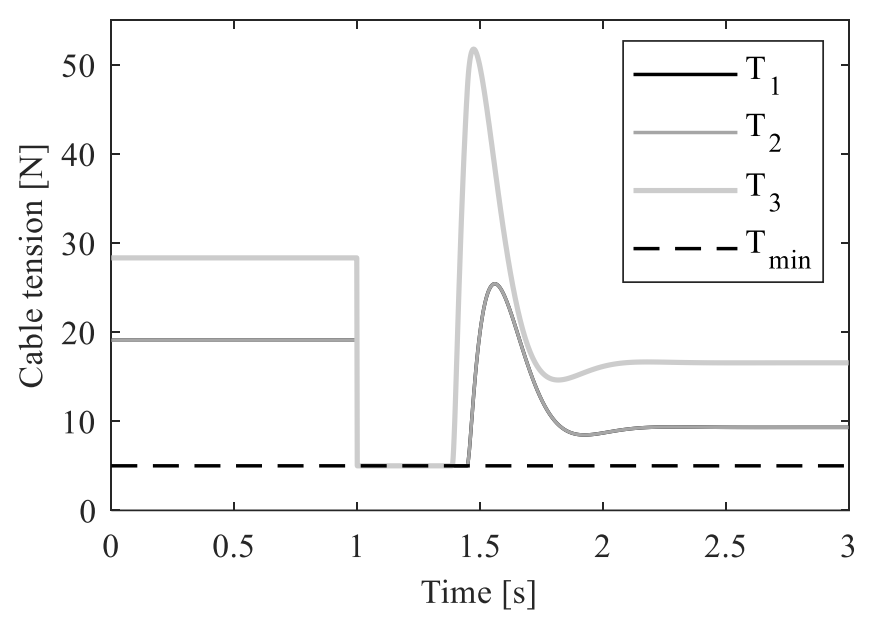

Figure 3. Cable tensions with step reference.

\subsubsection{Test 2: point-to-point motion with a feasible trajectory.}

To verify the control performance in the presence of a feasible trajectory, a $5^{\text {th }}$-degree polynomial motion law has been designed for a rest-to-rest motion lasting 3 seconds, to track a spatial straight line. Additional interval times of 1 second are also considered both at the beginning and at the end, in order to evaluate the controller in steady-state conditions. The spatial path tracking response is reported in Figure 4, together with its contour error in order to assess the performance of the proposed control algorithm. The control effectiveness is clearly proven, as the RMS value of the contour error is just $5.6 \times 10^{-5}[\mathrm{~m}]$. The error could be further reduced through finer tuning of the controller, which is however beyond the paper goals. The optimal cable tensions computed by the MPC are reported in Figure 5. 

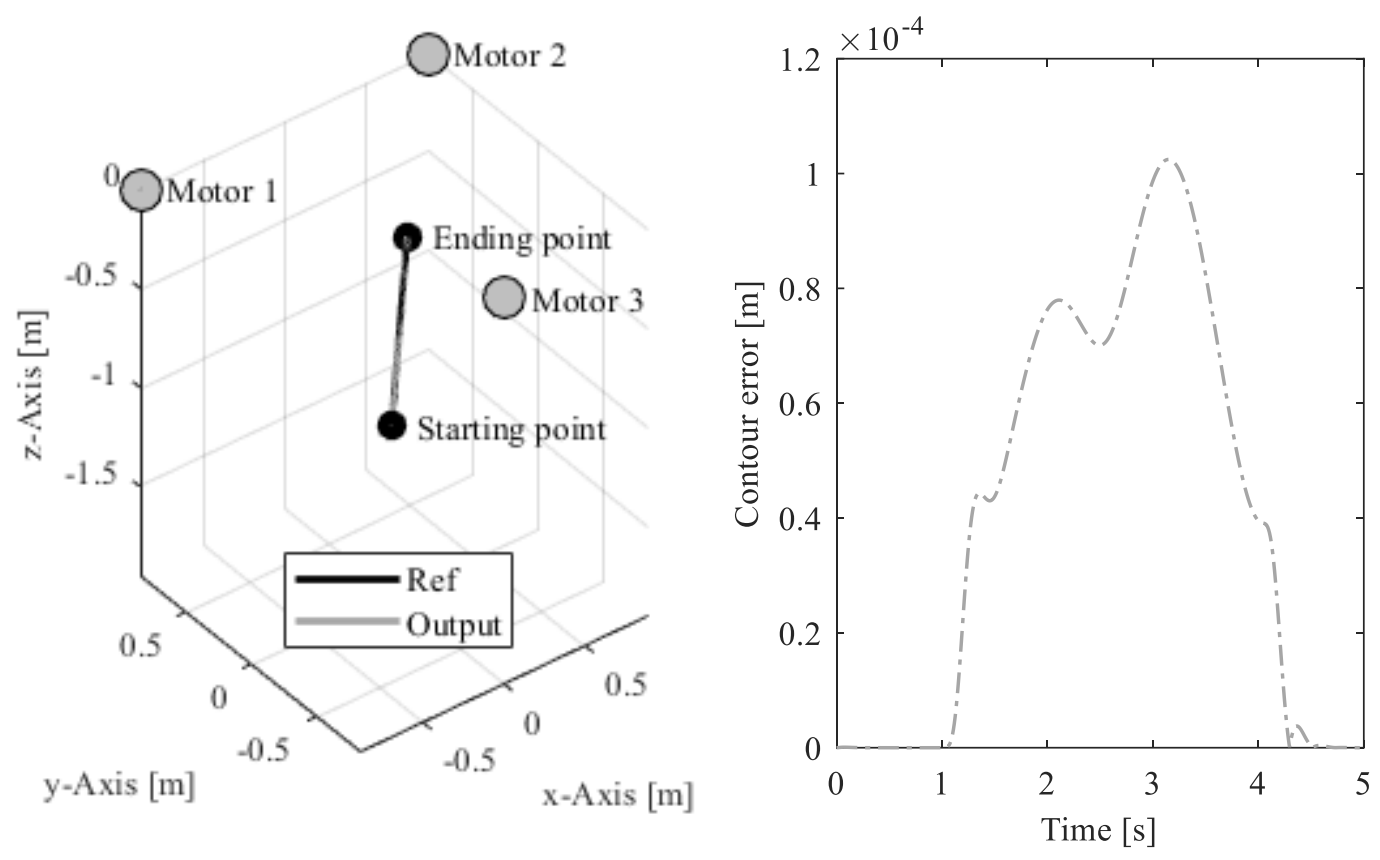

Figure 4. 3D-cartesian trajectory tracking response with $5^{\text {th }}$-degree polynomial reference (on the left) and its contour error (on the right).

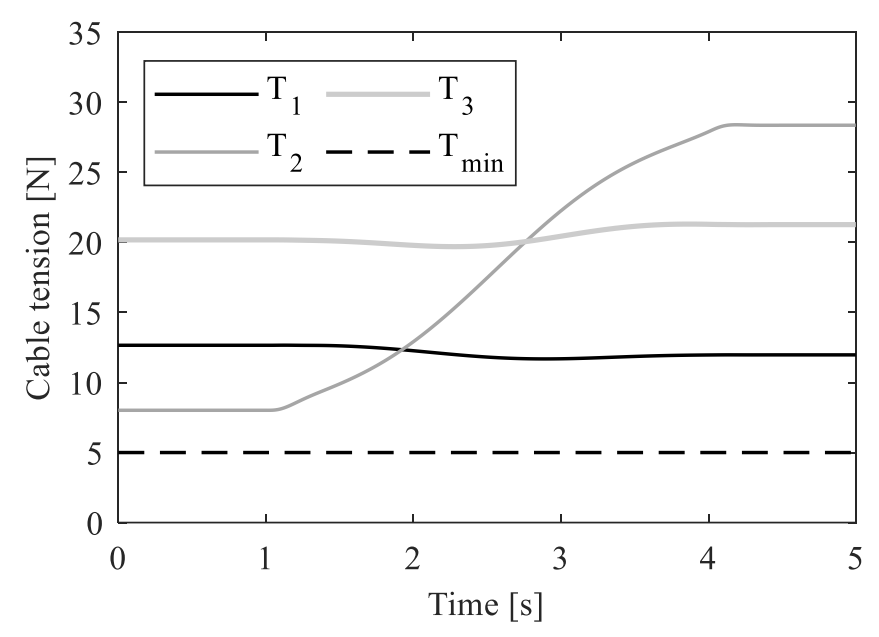

Figure 5. Cable tensions with $5^{\text {th }}$-degree polynomial reference.

\subsubsection{Test 3: circular path.}

The last test consists of a circular planar reference path to be tracked by the end-effector, considering an overall time interval of 5 seconds. The timing law adopted to parametrize the path is a $5^{\text {th }}$-degree polynomial, and the reference path goes outside the static workspace, which is a condition rarely considered in the literature, that makes the test case severe. In this test case a classical MPC formulation, without embedded integrator, is also tested (which is an easier and more common algorithm) in order to compare it with the MPC-EI; the same design parameters of Table 1 are used for both controllers, with the focus to make a fair comparison. Only the parameter $\mathbf{R}_{\Delta \mathrm{T}}$ is assumed with a different value; indeed, to avoid the instability of the classical MPC algorithm while keeping the same prediction and control horizons, its value is set one hundred times smaller and therefore equals to $1 \times 10^{-5} \mathbf{I}_{3}$. The planar tracking responses are displayed in Figure 6 for both controllers, together with their contour errors to have a clearer image of the performances. It should be noted that the classical MPC formulation, without embedded integrator, leads to a worse path tracking characterized by a huge contour error and a non-zero steady-state error. More precisely, the MPC algorithm without embedded integrator 
has an RMS contour error equals to $10.1 \times 10^{-3}[\mathrm{~m}]$, while the MPC-EI formulation provides a contour error whose RMS value is equal to $8.3 \times 10^{-4}[\mathrm{~m}]$. Since the contour error results to be lowered by one order of magnitude, these results corroborates the effectiveness of the presented controller. Finally, the optimal cable tensions commanded by the MPC-EI are shown in Figure 7 , underlying that this controller is capable to increase the overall performance in terms of path tracking, ensuring at the same time that the boundaries on cable tensions are verified.
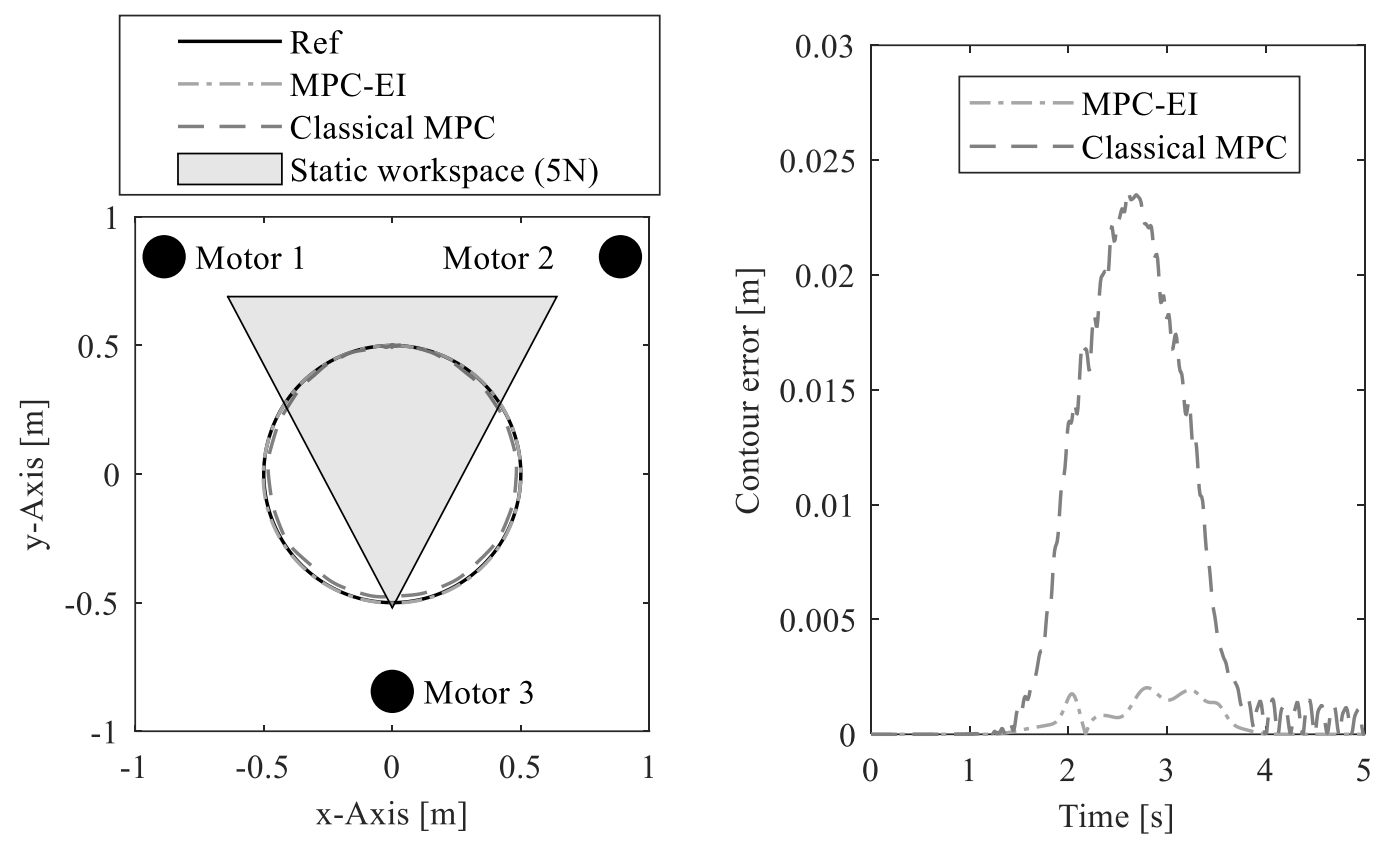

Figure 6. Comparison between Classical MPC and MPC-EI: 2D-cartesian trajectory tracking responses with circular reference (on the left) and relative contour errors (on the right).

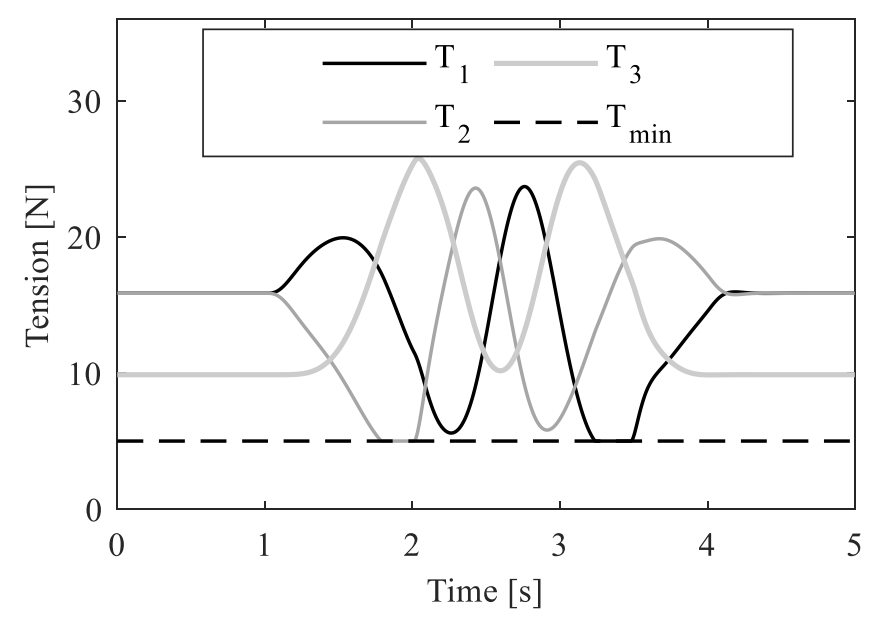

Figure 7. MPC-EI cable tensions with circular reference.

\section{CONCLUSIONS}

This paper proposes the preliminary results on a new Model Predictive Control algorithm tailored for path tracking control in Cable-Driven Robots. Control is performed by two sequential actions, that have been conceived to handle the highly nonlinear differential equations form the motor torque to the end-effector position. The first one is responsible for the evaluation of the optimal cable tensions while the second one consists in computing the required motor torques.By means of the implementation of a constrained time-varying MPC, the first step computes positive and bounded cable tensions, while minimizing a proper performance 
index that includes the tracking error and the tension variation. The use of an embedded integrator has been proposed to ensure accurate path tracking, in particular at steady-state conditions. The second action of the control scheme, on the other hand, permits to evaluate the necessary motor torques through a feedforward approach.

Three numerical test-cases have been proposed through a three-cable spatial robot, showing good performances in path tracking tasks and ensuring the positiveness of the cable tensions. To better understand the advantages coming from the MPC-EI, this latter has been also compared with a classical MPC formulation, without embedded integrator, which represents an easier and more common algorithm. By looking at the contour error, it has been noticed that MPC-EI has been capable to reduce it by one order of magnitude, ensuring at the same time the verification of the boundaries on all cable tensions and, therefore, confirming its supremacy.

\section{REFERENCES}

[1] Trevisani, A.: Underconstrained planar cable-direct-driven robots: A trajectory planning method ensuring positive and bounded cable tensions. Mechatronics 20 (2010) 113-27 doi:10.1016/j.mechatronics.2009.09.011

[2] Idà, E., Bruckmann, T., Carricato, M.: Rest-to-Rest Trajectory Planning for Underactuated CableDriven Parallel Robots. IEEE Trans. Robot. 35 (2019) 1338-51 doi:10.1109/TRO.2019.2931483

[3] Korayem, M. H., Tourajizadeh, H., Bamdad, M.: Dynamic load carrying capacity of flexible cable suspended robot: Robust feedback linearization control approach. J. Intell. Robot. Syst. Theory Appl. 60 (2010) 341-63 doi:10.1007/s10846-010-9423-x

[4] Khosravi, M. A., Taghirad, H. D.: Robust PID control of fully-constrained cable driven parallel robots. Mechatronics 24 (2014) 87-97 doi:10.1016/j.mechatronics.2013.12.001

[5] Boscariol, P., Gasparetto, A., Zanotto, V.: Active position and vibration control of a flexible links mechanism using model-based predictive control. J. Dyn. Syst. Meas. Control. Trans. ASME 132 (2010) 1-4 doi:10.1115/1.4000658

[6] Boscariol, P., Gasparetto, A., Zanotto, V.: Simultaneous position and vibration control system for flexible link mechanisms. Meccanica 46 (2011) 723-37 doi:10.1007/s11012-010-9333-9

[7] Boscariol, P., Zanotto, V.: Design of a controller for trajectory tracking for compliant mechanisms with effective vibration suppression. Robotica 30 (2012) 15-29 doi:10.1017/S0263574711000415

[8] Vermillion, C., Sun, J., Butts, K.: Model predictive control allocation for overactuated systems Stability and performance. In: IEEE Conference on Decision and Control, New Orleans, LA, USA (2007) 1251-6 doi:10.1109/CDC.2007.4434722

[9] Katliar, M., Fischer, J., Frison, G., Diehl, M., Teufel, H., Bülthoff, H. H.: Nonlinear Model Predictive Control of a Cable-Robot-Based Motion Simulator. IFAC-PapersOnLine 50 (2017) 9833-9 doi:10.1016/j.ifacol.2017.08.901

[10] Qi, R., Rushton, M., Khajepour, A., Melek, W. W.: Decoupled modeling and model predictive control of a hybrid cable-driven robot (HCDR). Rob. Auton. Syst. 118 (2019) 1-12 doi:10.1016/j.robot.2019.04.013

[11] Santos, J. C., Chemori, A., Gouttefarde, M.: Model predictive control of large-dimension cabledriven parallel robots. Mech. Mach. Sci. 74 (2019) 221-32 doi:10.1007/978-3-030-20751-9_19

[12] Santos, J. C., Chemori, A., Gouttefarde, M.: Redundancy Resolution Integrated Model Predictive Control of CDPRs: Concept, Implementation and Experiments. In: IEEE International Conference on Robotics and Automation (ICRA), Paris, France (2020) 3889-95 doi:10.1109/ICRA40945.2020.9197271

[13] Xiang, S., Gao, H., Liu, Z., Gosselin, C.: Dynamic transition trajectory planning of three-DOF cablesuspended parallel robots via linear time-varying MPC. Mech. Mach. Theory 146 (2020) 103715 doi:10.1016/j.mechmachtheory.2019.103715

[14] Richiedei, D., Tamellin, I., Trevisani, A.: A homotopy transformation method for interval-based model updating of uncertain vibrating systems. Mech. Mach. Theory $160 \quad$ (2021) 104288 doi:10.1016/j.mechmachtheory.2021.104288

[15] Idà, E., Briot, S., Carricato, M.: Identification of the inertial parameters of underactuated Cable$\begin{array}{lllllll}\text { Driven Parallel Robots. Mech. Mach. Theory } 167 & \text { (2022) } & 104504\end{array}$ doi:10.1016/j.mechmachtheory.2021.104504 